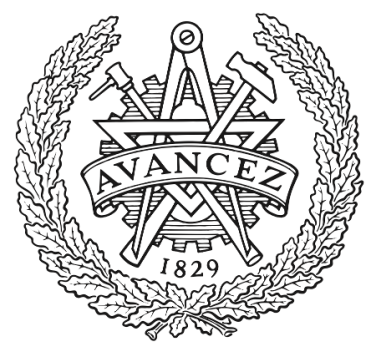

CHALMERS

UNIVERSITY OF TECHNOLOGY

\title{
Low-Complexity 5G Slam with CKF-PHD Filter
}

Downloaded from: https://research.chalmers.se, 2023-04-26 10:15 UTC

Citation for the original published paper (version of record):

Kim, H., Granström, K., Kim, S. et al (2020). Low-Complexity 5G Slam with CKF-PHD Filter.

ICASSP, IEEE International Conference on Acoustics, Speech and Signal Processing - Proceedings, 2020-May: 5220-5224. http://dx.doi.org/10.1109/ICASSP40776.2020.9053132

N.B. When citing this work, cite the original published paper. 


\title{
LOW-COMPLEXITY 5G SLAM WITH CKF-PHD FILTER
}

\author{
Hyowon Kim ${ }^{\star} \quad$ Karl Granström ${ }^{\dagger} \quad$ Sunwoo Kim ${ }^{\star} \quad$ Henk Wymeersch $^{\dagger}$ \\ * Department of Electronics and Computer Engineering, Hanyang University, Seoul, Korea \\ $\dagger$ Department of Electrical Engineering, Chalmers University of Technology, Sweden
}

\begin{abstract}
In $5 \mathrm{G}$ mmWave, simultaneous localization and mapping (SLAM) allows devices to exploit map information to improve their position estimate. Even the most basic SLAM filter based on a Rao-Blackwellized particle filter (RBPF) combined with a probability hypothesis density (PHD) map representation exhibits high complexity. This paper proposes a new implementation method for the 5G SLAM using message passing (MP) and the cubature Kalman filter (CKF). We demonstrate that the proposed method significantly reduces the complexity while retaining the SLAM accuracy of the RBPF-PHD approach.
\end{abstract}

Index Terms - 5G mmWave, CKF, cooperative SLAM, message passing, multi-model PHD.

\section{INTRODUCTION}

5G mmWave positioning systems benefit from large bandwidths and large antenna arrays which respectively lead to high resolution in both time and angle domain [1]. In addition, the non-line-of-sight (NLOS) signal paths are useful for both localization [1,2], and simultaneous localization and mapping (SLAM) of the physical environment [3-6]. 5G SLAM methods have been developed based on geometry [7, 8], message passing (MP) [3-5], and random finite set (RFS) theory [6]. The RFS methods have the benefit that they can easily handle an unknown number of objects, clutter measurements, and uncertainty of data association between objects and measurements [9], however, RFS methods also come with a relatively higher computational cost. In contrast, MP methods are a compromise between performance and computational complexity and can deal with unknown data association, however, MP methods deal less easily with clutter and an unknown number of objects. Among RFS SLAM methods, the Rao-Blackwellized particle filter (RBPF) probability hypothesis density (PHD) is widely used, as it avoids explicit enumeration of the data associations [10,11]. In [6], such a RBPF-PHD was applied to the 5G SLAM problem, showing how a vehicle can map a propagation environment comprising of reflecting surfaces and small scattering point objects. In [12], MP combined with RFS was proposed for low-complexity joint sensor and target tracking.

In this paper, we propose a low-complexity 5G SLAM

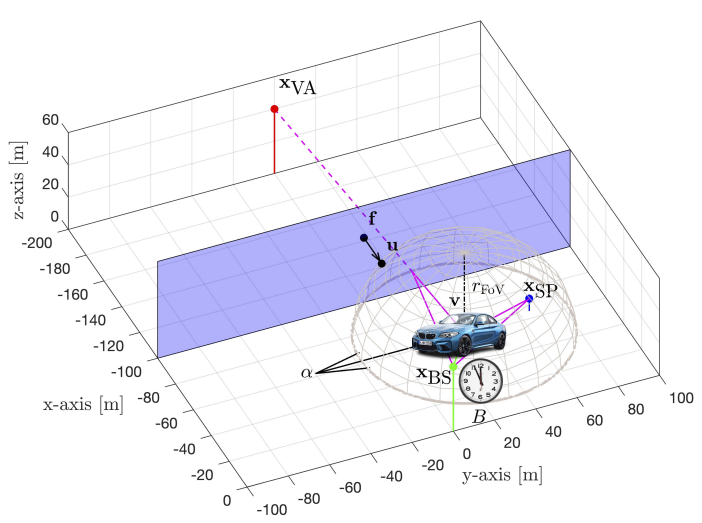

Fig. 1. Vehicle scenario with the vehicle (heading $\alpha$ shown with an arrow and clock bias $B$ ) and environment (BS, VA, and SP). A half-sphere with radius $r_{\text {FoV }}$ indicates the SP FoV of the vehicle.

method applied to a vehicular scenario, combining the performance of the PHD filter with the low complexity of MP. In particular, we perform MP on a factor graph representation [13, 14] of the joint posterior density of vehicle trajectories and map, and determine the marginal posterior density of vehicle state and map state. To represent the vehicle state messages, we use the low-complexity cubature Kalman filter (CKF) [15] instead of the high-complexity particle representation.

\section{MODEL}

In this section, the vehicle environment and models are described, which are adopted and summarized from previous 5G SLAM works [4-6].

\subsection{Vehicle and Environment Model}

We consider an environment with a base station (BS), large reflecting surfaces, and small objects, and a moving vehicle (see Fig. 11. The BS has known location, each small object is characterized by an unknown scattering point (SP) location, and each reflecting surface by an unknown virtual anchor (VA) location. The different objects (i.e., the signal sources) in the environment are characterized by a type $m \in\{\mathrm{BS}, \mathrm{VA}, \mathrm{SP}\}$, 


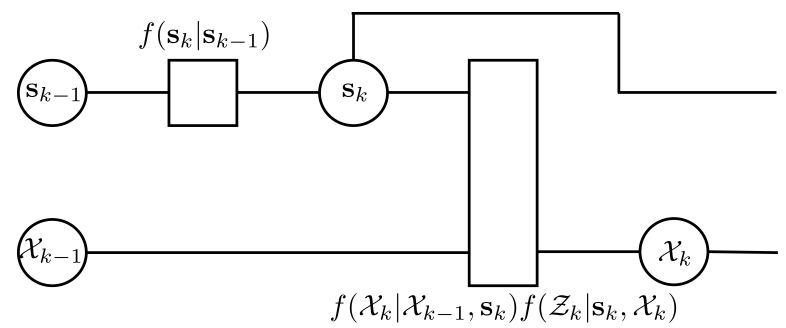

Fig. 2. Factor graph for representing one time step of the factorization of $f\left(\mathbf{s}_{0: k}, \mathcal{X}_{0: k} \mid \mathcal{Z}_{1: k}\right)$.

and and an object location $\mathbf{x}_{k} \in \mathbb{R}^{3}$. The vehicle state at time $k$ is denoted by $\mathbf{s}_{k}=\left[\mathbf{v}_{k}, \alpha_{k}, \zeta_{k}, \xi_{k}, B_{k}\right]^{\top} \in \mathbb{R}^{7}$, where $\mathbf{v}_{k}=\left[x_{v, k}, y_{v, k}, z_{v, k}\right]^{\top}, \alpha_{k}, \zeta_{k}, \xi_{k}$, and $B_{k}$ are respectively the location, heading, translation speed, turn-rate, and clock bias. The vehicle movement at time $k$ is modeled by a transition density $f\left(\mathbf{s}_{k} \mid \mathbf{s}_{k-1}\right)$ of the form $\mathbf{s}_{k}=\mathbf{v}\left(\mathbf{s}_{k-1}\right)+\mathbf{q}_{k}$, where $\mathrm{v}(\cdot)$ is a known transition function and $\mathbf{q}_{k} \sim \mathcal{N}\left(\mathbf{0}, \mathbf{Q}_{k}\right)$.

\subsection{Observation Model}

The BS periodically transmits a pilot signal, which is reflected by large surfaces and scattered by small objects, before it is received by the vehicle. Hence, the vehicle observes different signal paths coming from different object types. With each object we associate a detection probability $p_{\mathrm{D}, k}\left(\mathbf{s}_{k}, \mathbf{x}, m\right) \in$ $[0,1]$, depending on the field-of-view (FoV), visualized as a half-sphere in Fig. 1. We denote the measurements set a time $k$ by $\mathcal{Z}_{k}$ with elements $\mathbf{z}_{k, l} \in \mathbb{R}^{5}$, where $l$ indicates the signal path. We model the $\mathbf{z}_{k, l}$ for detected objects as

$$
\mathbf{z}_{k, l}=\mathrm{h}\left(\mathbf{s}_{k}, \mathbf{x}_{k}, m\right)+\mathbf{r}_{k, l},
$$

where $\mathrm{h}\left(\mathbf{s}_{k}, \mathbf{x}_{k}, m\right)=\left[\tau_{k, l}, \boldsymbol{\theta}_{k, l}^{\top}, \boldsymbol{\phi}_{k, l}^{\top}\right]^{\top}$ and measurement noise $\mathbf{r}_{k, l} \sim \mathcal{N}\left(\mathbf{0}, \mathbf{R}_{k, l}\right)$. Here, $\tau_{k, l}, \boldsymbol{\theta}_{k, l}$, and $\phi_{k, l}$ denote the time-of-arrival (TOA), direction-of-arrival (DOA), and direction-of-departure (DOD), respectively, computed from the geometric relations in [4, 6] for $m$. In addition, there may be false alarms in the form of clutter measurements $\mathbf{z}_{k, l}$, generated according to a clutter intensity $c(\mathbf{z})$.

\section{MULTI-MODEL PHD FILTER WITH MP}

The vehicle has a prior density $f\left(\mathbf{s}_{0}\right)$, and the known location of the BS. Based on the the sets measurements $\mathcal{Z}_{k}$ at every time step $k$, the problem considered in this paper is to estimate the vehicle state and build up the map. To this end, we define the RFS of the objects $\mathcal{X}_{k}$, comprising both the location and type, with density $f\left(\mathcal{X}_{k}\right)$.

\subsection{Factorization}

We denote $f\left(\mathbf{s}_{0: K}, \mathcal{X}_{0: K} \mid \mathcal{Z}_{1: K}\right)$ the full joint posterior density, which can be factorized for sequential estimation:

$$
\begin{aligned}
& f\left(\mathbf{s}_{0: K}, \mathcal{X}_{0: K} \mid \mathcal{Z}_{1: K}\right) \propto \\
& f\left(\mathbf{s}_{0}\right) f\left(\mathcal{X}_{0}\right) \prod_{k=1}^{K} f\left(\mathbf{s}_{k} \mid \mathbf{s}_{k-1}\right) f\left(\mathcal{X}_{k} \mid \mathcal{X}_{k-1}, \mathbf{s}_{k}\right) f\left(\mathcal{Z}_{k} \mid \mathbf{s}_{k}, \mathcal{X}_{k}\right),
\end{aligned}
$$

where $f\left(\mathcal{X}_{k} \mid \mathcal{X}_{k-1}, \mathbf{s}_{k}\right)$ is the object transition density (which depends on the vehicle state, since new static objects will become visible when they are in proximity of the vehicle) and $f\left(\mathcal{Z}_{k} \mid \mathbf{s}_{k}, \mathcal{X}_{k}\right)$ is the set likelihood function. The corresponding factor graph is shown in Fig. 2. We note that the map transition factor $f\left(\mathcal{X}_{k} \mid \mathcal{X}_{k-1}, \mathbf{s}_{k}\right)$ is integrated with the likelihood factor $f\left(\mathcal{Z}_{k} \mid \mathbf{s}_{k}, \mathcal{X}_{k}\right)$ by MP rule, abbreviated by $\Psi\left(\mathbf{s}_{k}, \mathcal{X}_{k}\right)$.

\subsection{Message Passing and Scheduling}

We denote the vehicle and map beliefs at time $k-1$ by $b_{k-1}\left(\mathbf{s}_{k-1}\right)$ and $b_{k-1}\left(\mathcal{X}_{k-1}\right)$, respectively. The message passing and its scheduling for calculating the new beliefs at time $k$ are described.

\subsubsection{Prediction}

The variable $\mathbf{s}_{k-1}$ sends its belief $b_{k-1}\left(\mathbf{s}_{k-1}\right)$ to the factor $f\left(\mathbf{s}_{k} \mid \mathbf{s}_{k-1}\right)$. Then, a vehicle prediction message $b_{k \mid k-1}\left(\mathbf{s}_{k}\right)$ is calculated as

$$
b_{k \mid k-1}\left(\mathbf{s}_{k}\right) \propto \int b_{k-1}\left(\mathbf{s}_{k-1}\right) f\left(\mathbf{s}_{k} \mid \mathbf{s}_{k-1}\right) \mathrm{ds}_{k-1},
$$

and the variable $\mathbf{s}_{k}$ sends the calculated message $b_{k \mid k-1}\left(\mathbf{s}_{k}\right)$ to the factor $\Psi\left(\mathbf{s}_{k}, \mathcal{X}_{k}\right)$. Similarly, the variable $\mathcal{X}_{k-1}$ sends the belief $b_{k-1}\left(\mathcal{X}_{k-1}\right)$ to the factor $\Psi\left(\mathbf{s}_{k}, \mathcal{X}_{k}\right)$. For considering detected objects at time $k$ and the FoV in the map prediction, we introduce an auxiliary message

$$
v_{k}\left(\mathcal{X}_{k} \mid \mathbf{s}_{k}\right) \propto \int b_{k-1}\left(\mathcal{X}_{k-1}\right) f\left(\mathcal{X}_{k} \mid \mathcal{X}_{k-1}, \mathbf{s}_{k}\right) \delta \mathcal{X}_{k-1} .
$$

\subsubsection{Correction}

The updated belief of $\mathbf{s}_{k}$ is given by the product of the prediction (3) and the message from the factor $\Psi\left(\mathbf{s}_{k}, \mathcal{X}_{k}\right)$ :

$$
b_{k}\left(\mathbf{s}_{k}\right) \propto b_{k \mid k-1}\left(\mathbf{s}_{k}\right) \int v_{k}\left(\mathcal{X}_{k} \mid \mathbf{s}_{k}\right) f\left(\mathcal{Z}_{k} \mid \mathbf{s}_{k}, \mathcal{X}_{k}\right) \delta \mathcal{X}_{k} .
$$

The updated belief of $\mathcal{X}_{k}$ is the message from $\Psi\left(\mathbf{s}_{k}, \mathcal{X}_{k}\right)$ :

$$
b_{k}\left(\mathcal{X}_{k}\right) \propto \int b_{k \mid k-1}\left(\mathbf{s}_{k}\right) v_{k}\left(\mathcal{X}_{k} \mid \mathbf{s}_{k}\right) f\left(\mathcal{Z}_{k} \mid \mathbf{s}_{k}, \mathcal{X}_{k}\right) \mathbf{d s}_{k} .
$$

We observe that both the prediction and the correction involve set integrals and representation of set densities. In the next section, we will describe a low-complexity approximate implementation of these MP rules.

\subsection{Message Implementation}

\subsubsection{PHD and Gaussian approximation}

We will approximate the RFS densities with their PHDs and then show how to compute the predicted and corrected PHDs as well as the predicted and corrected vehicle beliefs, based on the PHDs. We denote the PHD for object type $m$ at time $k$ by $D_{k}(\mathbf{x}, m)$ and represent it by a Gaussian mixture (GM). 
We initialize $D_{0}(\mathbf{x}, m)=0$ for object type $m=\{\mathrm{VA}, \mathrm{SP}\}$ and $D_{k}(\mathbf{x}, \mathrm{BS})=\delta\left(\mathbf{x}-\mathbf{x}_{\mathrm{BS}}\right)$. Similarly, we approximate $b_{k}\left(\mathbf{s}_{k}\right)$ with a Gaussian density $\mathcal{N}\left(\mathbf{s} ; \hat{\mathbf{s}}_{k}, \hat{\mathbf{U}}_{k}\right)$, and initialize $b_{0}\left(\mathbf{s}_{0}\right)=f\left(\mathbf{s}_{0}\right)$.

\subsubsection{Prediction}

1) Vehicle 3): Given $b_{k-1}\left(\mathbf{s}_{k-1}\right)=\mathcal{N}\left(\mathbf{s} ; \hat{\mathbf{s}}_{k-1}, \hat{\mathbf{U}}_{k-1}\right)$, we decompose $\hat{\mathbf{U}}_{k-1}=\mathbf{G G}^{\top}$ and generate cubature points (CPs) by

$$
\mathbf{s}_{k-1}^{c}=\mathbf{G} \epsilon_{\mathbf{s}_{k}}^{c}+\hat{\mathbf{s}}_{k-1}, \quad c=1, \ldots, 2 \mathrm{~d}\left(\mathbf{s}_{k}\right)
$$

The vehicle CP for all $c$ is propagated as $\dot{\mathbf{s}}_{k}^{c}=\mathrm{v}\left(\mathbf{s}_{k-1}^{c}\right)$, and then the predicted mean and covariance are calculated as

$\overline{\mathbf{s}}_{k}=\frac{1}{2 \mathrm{~d}(\mathbf{s})} \sum_{c=1}^{2 \mathrm{~d}(\mathbf{s})} \dot{\mathbf{s}}_{k}^{c}, \quad \overline{\mathbf{U}}_{k}=\frac{1}{2 \mathrm{~d}(\mathbf{s})} \sum_{c=1}^{2 \mathrm{~d}(\mathbf{s})} \dot{\mathbf{s}}_{k}^{c} \dot{\mathbf{s}}_{k}^{c \top}-\overline{\mathbf{s}}_{k} \overline{\mathbf{s}}_{k}^{\top}+\mathbf{Q}_{k}$.

2) Map 47: We generate the $\mathrm{CP}$ set $\left\{\overline{\mathbf{s}}_{k}^{c}, \bar{w}_{k}^{c}\right\}_{c=1}^{2 \mathrm{~d}\left(\mathbf{s}_{k}\right)}$ with weights $w_{k-1}^{c}=1 /\left(2 \mathrm{~d}\left(\mathbf{s}_{k}\right)\right)$, which is from $\mathcal{N}\left(\mathbf{s} ; \overline{\mathbf{s}}_{k}, \overline{\mathbf{U}}_{k}\right)$ similar to (7). Given $D_{k-1}(\mathbf{x}, m)$, the predicted map PHD corresponding to $v_{k}\left(\mathcal{X}_{k} \mid \mathbf{s}_{k}\right)$ in (4) is implemented as

$$
D_{k \mid k-1}\left(\mathbf{x}, m \mid \overline{\mathbf{s}}_{k}^{c}\right)=D_{k-1}(\mathbf{x}, m)+D_{k}^{b}\left(\mathbf{x}, m \mid \overline{\mathbf{s}}_{k}^{c}\right),
$$

where $D_{k}^{b}\left(\mathbf{x}, m \mid \overline{\mathbf{s}}_{k}^{c}\right)$ is a birth ${ }^{2}$ intensity (also a PHD), modeled as a GM. Hence, $D_{k \mid k-1}\left(\mathbf{x}, m \mid \overline{\mathbf{s}}_{k}^{c}\right)$ is also a GM, conditioned on the $\mathrm{CP} \overline{\mathbf{s}}_{k}^{c}$ :

$$
D_{k \mid k-1}\left(\mathbf{x}, m \mid \overline{\mathbf{s}}_{k}^{c}\right)=\sum_{j=1}^{\bar{J}_{k}^{c}(m)} \bar{\gamma}_{k}^{c, j}(m) \mathcal{N}\left(\mathbf{x} ; \overline{\mathbf{x}}_{k}^{c, j}(m), \overline{\mathbf{P}}_{k}^{c, j}(m)\right)
$$

\subsubsection{Correction}

In the update, we begin by computing CPs for the vehicle state. For each $\mathrm{CP}$, we compute the corresponding updated PHD. To update the vehicle state with the standard CKF update, a predicted measurement is necessary. However, due to the random nature of the set of measurements, it is difficult to compute a predicted set of measurements in a straightforward way. Instead, we use the following computationally efficient alternative. For each CP, we compute the posterior weight as in (11). The posterior weights are normalized, and posterior mean and covariance for the vehicle state are computed as the sample mean and sample covariance.

\footnotetext{
${ }^{1}$ For the CPs, we define $\mathrm{d}(\star)$ as the number of unknown variables of $\star$, and $\epsilon_{\star}^{c}$ is indicated by the $c$-th column vector of the matrix $\sqrt{\mathrm{d}(\star)}\left[\mathbf{I}^{\mathrm{d}(\star)},-\mathbf{I}^{\mathrm{d}(\star)}\right] \in \mathbb{R}^{\mathrm{d}(\star) \times 2 \mathrm{~d}(\star)}$, where $\mathbf{I}^{\mathrm{d}(\star)} \in \mathbb{R}^{\mathrm{d}(\star) \times \mathrm{d}(\star)}$ is the identity matrix.

${ }^{2}$ The births can be generated in different ways. We have used an adaptive birth density from [16], details of which are found in [6. Sec. III].
}

1) Vehicle (5): The belief $b_{k}\left(\mathbf{s}_{k}\right)$ is found by computing posterior weights for each $\mathrm{CP}$ as in [6, Appendix B], see also [17]:

$$
\tilde{w}_{k}^{c} \propto \bar{w}_{k}^{c} \prod_{\mathbf{z} \in \mathcal{Z}_{k}}\left\{c(\mathbf{z})+\sum_{m} \int \nu\left(\mathbf{z}, \mathbf{x}, m, \overline{\mathbf{s}}_{k}^{c}\right) \mathrm{d} \mathbf{x}\right\}
$$

where $\nu\left(\mathbf{z}, \mathbf{x}, m, \overline{\mathbf{s}}_{k}^{c}\right)=\sum_{j=1}^{\bar{J}_{k}^{c}(m)} \nu^{j}\left(\mathbf{z}, \mathbf{x}, m, \overline{\mathbf{s}}_{k}^{c}\right)$. Introducing $j(\mathbf{z})$ as the birth index $j$ corresponding to $\mathbf{z}$, then when $j(\mathbf{z})=$ $j$,

$$
\nu^{j}\left(\mathbf{z}, \mathbf{x}, m, \overline{\mathbf{s}}_{k}^{c}\right)=\bar{\gamma}_{k}^{c, j}(m) \mathcal{N}\left(\mathbf{x} ; \overline{\mathbf{x}}_{k}^{c, j}(m), \overline{\mathbf{P}}_{k}^{c, j}(m)\right),
$$

otherwise,

$$
\begin{aligned}
& \nu^{j}\left(\mathbf{z}, \mathbf{x}, m, \overline{\mathbf{s}}_{k}^{c}\right)=p_{\mathrm{D}, k}^{c, j}(m) \bar{\gamma}_{k}^{c, j}(m) \mathcal{N}\left(\mathbf{x} ; \hat{\mathbf{x}}_{k}^{c, j}(m), \hat{\mathbf{P}}_{k}^{c, j}(m)\right) \\
& \times \mathcal{N}\left(\mathbf{z} ; \mathrm{h}\left(\hat{\mathbf{s}}_{k}^{c}, \overline{\mathbf{x}}_{k}^{c, j}(m), m\right), \mathbf{S}_{\mathbf{z z}, k}^{c, j}(m)\right),
\end{aligned}
$$

where $p_{\mathrm{D}, k}^{c, j}(m)$ is an adaptive detection probability ${ }^{3}$ which is adopted from [6] to avoid decrease of the detected object weight. By a standard $\mathrm{CKF}$ update, the Gaussian density $\mathcal{N}\left(\mathbf{x} ; \hat{\mathbf{x}}_{k}^{c, j}(m), \hat{\mathbf{P}}_{k}^{c, j}(m)\right)$ and corresponding constant $\mathcal{N}\left(\mathbf{z} ; \mathbf{h}\left(\overline{\mathbf{s}}_{k}^{c}, \overline{\mathbf{x}}_{k}^{c, j}(m), m\right), \mathbf{S}_{\mathbf{z z}, k}^{c, j}(m)\right)$ are calculated. The weight is normalized as

$$
\hat{w}_{k}^{c}=\frac{\tilde{w}_{k}^{c}+\eta}{\sum_{c=1}^{2 \mathrm{~d}\left(\mathbf{s}_{k}\right)}\left(\tilde{w}_{k}^{c}+\eta\right)}
$$

where $\eta \geq 0$ is a tuning parameter to to avoid the problem of weight degeneracy. We set $\eta=1 / 2 \mathrm{~d}\left(\mathbf{s}_{\mathbf{k}}\right)$. Then, $\hat{\mathbf{s}}_{k}$ and $\hat{\mathbf{U}}_{k}$ are respectively calculated as

$$
\hat{\mathbf{s}}_{k}=\sum_{c=1}^{2 \mathrm{~d}\left(\mathbf{s}_{k}\right)} \hat{w}_{k}^{c} \overline{\mathbf{s}}_{k}^{c}, \quad \hat{\mathbf{U}}_{k}=\sum_{c=1}^{2 \mathrm{~d}\left(\mathbf{s}_{k}\right)} \hat{w}_{k}^{c}\left[\overline{\mathbf{s}}_{k}^{c}-\hat{\mathbf{s}}_{k}\right]\left[\overline{\mathbf{s}}_{k}^{c}-\hat{\mathbf{s}}_{k}\right]^{\top} .
$$

2) Map (6): Following (6), we first compute the conditional map PHD $D_{k}\left(\mathbf{x}, m \mid \overline{\mathbf{s}}_{k}^{c}\right)$ and then compute the expectation over $\mathbf{s}_{k}$ by using the weights computed in (14), used to represent $b_{k}\left(\mathbf{s}_{k}\right)$. We update the map PHD for each $c$ and $m$ [18]:

$$
\begin{aligned}
D_{k}\left(\mathbf{x}, m \mid \overline{\mathbf{s}}_{k}^{c}\right) & =D_{k \mid k-1}\left(\mathbf{x}, m \mid \overline{\mathbf{s}}_{k}^{c}\right)\left(1-p_{\mathrm{D}}\left(\mathbf{x}, m, \overline{\mathbf{s}}_{k}^{c}\right)\right) \\
& +\sum_{\mathbf{z} \in \mathcal{Z}_{k}} \frac{\nu^{c}\left(\mathbf{z}, \mathbf{x}, m, \overline{\mathbf{s}}_{k}^{c}\right)}{c(\mathbf{z})+\sum_{m^{\prime}} \int \nu^{c}\left(\mathbf{z}, \mathbf{x}^{\prime}, m^{\prime}, \overline{\mathbf{s}}_{k}^{c}\right) \mathrm{d} \mathbf{x}^{\prime}}
\end{aligned}
$$

The average PHD is $D_{k}(\mathbf{x}, m)=\sum_{c=1}^{2 \mathrm{~d}\left(\mathbf{s}_{k}\right)} \hat{w}_{k}^{c} D_{k}\left(\mathbf{x}, m \mid \overline{\mathbf{s}}_{k}^{c}\right)$, which is implemented using pruning and merging, described in [19, Table II].

\footnotetext{
${ }^{3}$ Given $\mathcal{N}\left(\mathbf{x} ; \overline{\mathbf{x}}_{k}^{c, j}(m), \overline{\mathbf{P}}_{k}^{c, j}(m)\right)$ with the highest density region $\mathcal{H}$, $p_{\mathrm{D}, k}^{c, j}(m)=\min _{\mathbf{x} \in \mathcal{H}} p_{\mathrm{D}, k}^{c, j}\left(\mathbf{x}, m, \overline{\mathbf{s}}_{k}^{c}\right)$. When $\mathcal{S}$ is the set of FoV region for $\overline{\mathbf{s}}_{k}^{c}, p_{\mathrm{D}, k}^{c, j}\left(\mathbf{x}, m, \overline{\mathbf{s}}_{k}^{c}\right)$ is calculated by $\int_{\mathcal{S}} \mathcal{N}\left(\mathbf{x} ; \overline{\mathbf{x}}_{k}^{c, j}(m), \overline{\mathbf{P}}_{k}^{c, j}(m)\right) \mathrm{d} \mathbf{x}$.
} 


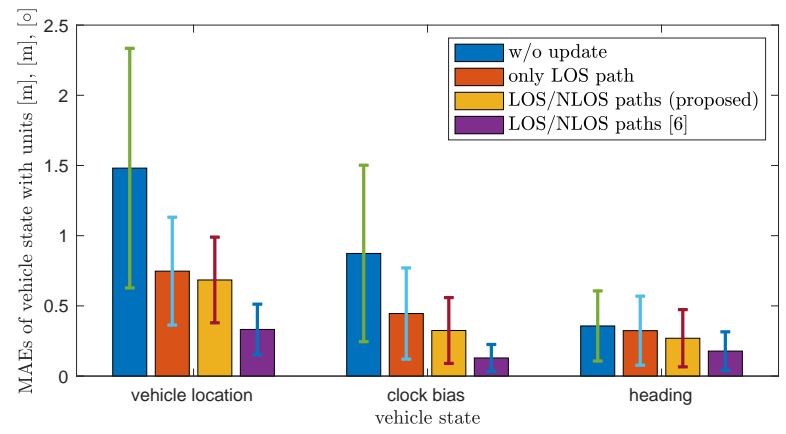

Fig. 3. MAEs and RMSE bars of the vehicle state (vehicle location, clock bias, and heading) by the proposed method compared to [6].

\section{NUMERICAL RESULTS}

\subsection{Simulation Setup}

We consider vehicle that moves along a circular road for $K=40$ with time interval 0.5 seconds, and the movement follows the mobility model [20, Chapter 5]. We set process noise covariance $\mathbf{Q}_{k}=\operatorname{diag}\left(\sigma_{x}^{2}, \sigma_{y}^{2}, 0, \sigma_{\alpha}^{2}, 0,0, \sigma_{B}^{2}\right)$, and $\sigma_{x}=0.2 \mathrm{~m}, \sigma_{y}=0.2 \mathrm{~m}, \sigma_{\alpha}=0.001 \mathrm{rad}, \sigma_{B}=0.2 \mathrm{~m}$, and $\mathbf{s}_{0}=[70.7285,0,0, \pi / 2,22.22, \pi / 10,300]^{\top}$, with units $\mathrm{m}, \mathrm{m}, \mathrm{m}, \mathrm{rad}, \mathrm{m} / \mathrm{s}, \mathrm{rad} / \mathrm{s}$, and $\mathrm{m}$. The prior $f\left(\mathrm{~s}_{0}\right)$ follows a Gaussian distribution, and the standard deviation is set to $[0.3,0.3,0,0.3,0,0,0.3]^{\top}$, with units as the vehicle state. We assume that the translation speed $\zeta_{k}$ and turn-rate $\xi_{k}$ are known. We set $\mathbf{R}_{k, l}=\operatorname{diag}\left(10^{-2}, 10^{-4}, 10^{-4}, 10^{-4}, 10^{-4}\right)$, with units $\mathrm{m}^{2}, \operatorname{rad}^{2}, \operatorname{rad}^{2}, \operatorname{rad}^{2}$, and $\operatorname{rad}^{2}$. When the predicted measurement covariance $\mathbf{S}_{\mathbf{z z}, k}^{c, j}(m)$ in 13 is calculate by the CKF, the measurement covariance $\mathbf{R}_{k, l}$ is replaced with $\mathbf{R}_{\text {up }}=9 \times \mathbf{R}_{k, l}$ to mitigate the effect of nonlinearity in the CKF.

The BS is located at $[0,0,40]^{\top} \mathrm{m}$. Four VAs are respectively located at $[200,0,40]^{\top},[-200,0,40]^{\top},[0,200,40]^{\top}$, $[0,-100,40]^{\top}$, with unit $\mathrm{m}$. Four SPs are respectively located at $\left[65,65, z_{\mathrm{SP}}\right]^{\top},\left[-65,65, z_{\mathrm{SP}}\right]^{\top},\left[-65,-65, z_{\mathrm{SP}}\right]^{\top}$, $\left[65,-65, z_{\mathrm{SP}}\right]^{\top}$, with unit $\mathrm{m}$, and $z_{\mathrm{SP}} \sim \mathcal{U}(0,40)$. We set the detection probability $p_{\mathrm{D}}=0.9$ within the FoV, the SP FoV $r_{\mathrm{FoV}}=50 \mathrm{~m}$ while VAs are always visible. In the the birth process, we set the birth weight $\gamma_{b, k}^{c, j}(m)=1.5 \times 10^{-5}$ for $m=\{\mathrm{VA}, \mathrm{SP}\}$. We consider clutter intensity $c(\mathbf{z})=$ $\lambda /\left(4 R_{\max } \pi^{4}\right)$ as the average of the number of clutter measurements (following Poisson distribution) $\lambda=1$, and the maximum sensing range $R_{\max }=200 \mathrm{~m}$. For detecting the object in the map PHD, we set the VA detection threshold $T_{\mathrm{VA}}=0.7$ and the SP detection threshold $T_{\mathrm{SP}}=0.55$. We use the average of the generalized optimal subpattern assignment (GOSPA) distance for measuring the mapping accuracy [21]. Simulation results were obtained by averaging over 10 Monte Carlo runs.

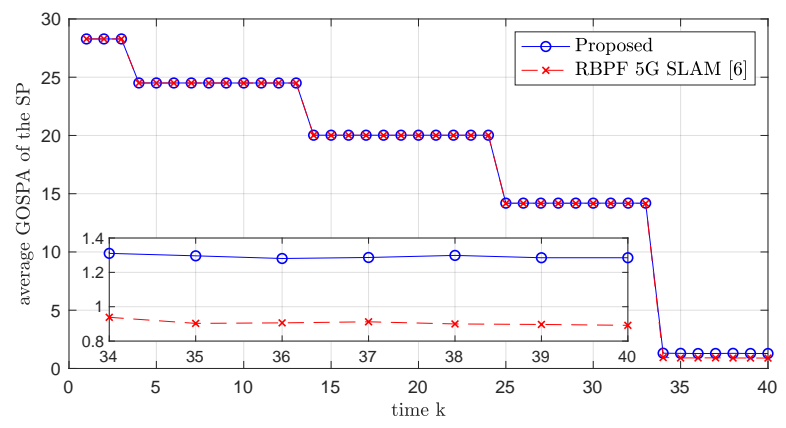

Fig. 4. Average GOSPA of SP by the proposed method compared to [6].

\subsection{Discussions}

Fig. 3 shows the mean square errors (MAEs) and root mean square error (RMSE) bars for estimating the vehicle location, clock bias, and heading with respect to the following four cases: i) vehicle prediction without the measurement update; ii) only using LOS path for the measurement update in the proposed PHD filter; iii) the proposed PHD filter; iv) the particle-based PHD filter without map fusion in [6]. By comparison of the results of the four cases, we confirmed that it is necessary for the measurement update to perform 5G SLAM, and using the NLOS measurements and mapping of the unknown physical environment improves the accuracy of the vehicle estimate.

Fig. 4 shows the average GOSPA of the SPs for the proposed method compared to [6]. The SP GOSPA decreases as the the vehicle progressively receives the scattered signals from all SPs. We confirmed that there is a slight degradation of the mapping in the proposed method compared to [6], but with a huge complexity gain: in terms of runtime, the proposed methods runs around 300 times faster than the RBPFPHD from [6].

Thus, the results clearly show that the proposed method can achieve significant gain in the complexity while sustaining the vehicle estimate accuracy.

\section{CONCLUSIONS}

We have proposed a novel 5G SLAM methods based on message passing and PHD filtering. The method exhibits similar performance to the more complex Rao-Blackwellized PHD filter, but with far lower complexity. This allows real-time processing in vehicles.

\section{Acknowledgments}

This work was supported, in part, by the MSIT (Ministry of Science and ICT), Korea, under the ITRC (Information Technology Research Center) support program (IITP-2019-2017-0-01637) supervised by the IITP (Institute for Information \& Communications Technology Planning \& Evaluation), by the Samsung Research Funding and Incubation Center of Samsung Electronics under Project Number SRFC-IT-1601-09, and the Swedish Research Council under grant No. 2018-03701. 


\section{REFERENCES}

[1] H. Wymeersch, G. Seco-Granados, G. Destino, D. Dardari, and F. Tufvesson, "5G mm-Wave positioning for vehicular networks," IEEE Wireless Commun., vol. 24, no. 6, pp. 80-86, Dec. 2018.

[2] K. Witrisal et al., "High-accuracy localization for assisted living: $5 \mathrm{G}$ systems will turn multipath channels from foe to friend," IEEE Signal Process. Mag., vol. 33, no. 2, pp. 59-70, Mar. 2016.

[3] R. Mendrzik, H. Wymeersch, and G. Bauch, "Joint localization and mapping through millimeter wave MIMO in 5G systems-extended version," arXiv preprint arXiv:1804.04417, 2018.

[4] H. Wymeersch, N. Garcia, H. Kim, G. Seco-Granados, S. Kim, F. Wen, and M. Fröhle, "5G mmWave downlink vehicular positioning," in Proc. IEEE Global Commun. Conf. (GLOBECOM), Abu Dhabi, UAE, Dec. 2018, pp. 206-212.

[5] H. Kim, H. Wymeersch, N. Garcia, G. Seco-Granados, and S. Kim, "5G mmWave vehicular tracking," in Proc. IEEE 52nd Asilomar Conf. Signals, Syst., Comput., Pacific Grove, CA, USA, Oct. 2018, pp. 541-547.

[6] H. Kim, K. Granström, L. Gao, G. Battistelli, S. Kim, and $\mathrm{H}$. Wymeersch, "5G mmWave cooperative positioning and mapping using multi-model PHD," arXiv preprint arXiv:1907.09806, 2019.

[7] A. Yassin, Y. Nasser, A. Y. Al-Dubai, and M. Awad, "MOSAIC: Simultaneous localization and environment mapping using mmWave without a-priori knowledge," IEEE Access, vol. 6, pp. 68932-68947, Nov. 2018.

[8] M. Aladsani, A. Alkhateeb, and G. C. Trichopoulos, "Leveraging mmWave imaging and communications for simultaneous localization and mapping," in Proc. 2019 IEEE Int. Conf. Acoust., Speech Signal Process. (ICASSP), Brighton, UK, May 2019, pp. 4539-4543.

[9] H. Durrant-Whyte and T. Bailey, "Simultaneous localization and mapping: Part I," IEEE Robot. Autom. Mag., vol. 13, no. 2, pp. 99-110, Jun. 2006.

[10] R. Mahler, Statistical multisource-multitarget information fusion, Artech House, Norwood, MA, USA, 2007.

[11] J. Mullane, B-N Vo, M. D. Adams, and B-T Vo, “A random-finite-set approach to Bayesian SLAM," IEEE Trans. Robot., vol. 27, no. 2, pp. 268-282, Apr. 2011.

[12] M. Fröhle, C. Lindberg, K. Granström, and H. Wymeersch, "Multisensor poisson multi-bernoulli filter for joint target-sensor state tracking," IEEE Trans. Intell. Veh., vol. 4, no. 4, pp. 609-621, Aug. 2019.
[13] H-A Loeliger, "An introduction to factor graphs," IEEE Signal Process. Mag., vol. 21, no. 1, pp. 28-41, Jan. 2004.

[14] F. R. Kschischang, B. J. Frey, and H-A Loeliger, "Factor graphs and the sum-product algorithm," IEEE Trans. Inf. Theory, vol. 47, no. 2, pp. 498-519, Feb. 2001.

[15] I. Arasaratnam and S. Haykin, "Cubature Kalman filters," IEEE Trans. Autom. Control, vol. 54, no. 6, pp. 1254-1269, Jun. 2009.

[16] B. Ristic, D. Clark, B-N Vo, and B-T Vo, "Adaptive target birth intensity for PHD and CPHD filters," IEEE Trans. Aerosp. Electron. Syst., vol. 48, no. 2, pp. 16561668, Apr. 2012.

[17] Á. F. García-Fernández, J. L. Williams, K. Granström, and L. Svensson, "Poisson multi-Bernoulli mixture filter: Direct derivation and implementation," IEEE Trans. Aerosp. Electron. Syst., vol. 54, no. 4, pp. 1883-1901, Aug. 2018.

[18] R. P. S. Mahler, "Multitarget Bayes filtering via firstorder multi target moments," IEEE Trans. Aerosp. Electron. Syst., vol. 39, no. 4, pp. 1152-1178, Oct. 2003.

[19] B-N Vo and W-K Ma, "The Gaussian mixture probability hypothesis density filter,' IEEE Trans. Signal Process., vol. 54, no. 11, pp. 4091-4104, Nov. 2006.

[20] S. Thrun, W. Burgard, and D. Fox, Probabilistic Robotics (Intelligent Robotics and Autonomous Agents Series), MIT Press, 2005.

[21] A. S. Rahmathullah, Á. F. García Fernández, and L. Svensson, "Generalized optimal sub-pattern assignment metric," in Proc. 20th Int. Conf. Inf. Fusion (FUSION), Xian, China, Jul. 2017, pp. 1-8. 